\title{
SAFETY DATA SHEET
}

\section{Identification}

Product identifier

Other means of identification

Recommended use

Recommended restrictions
Titanium carbide compound aqueous solution

None.

Not available.

None known.

Manufacturer/Importer/Supplier/Distributor information

Manufacturer Name

Address

Department 1

Contact person

Phone call

E-mail

Emergency person

\section{Hazard(s) identification}

Physical hazards
Health hazards
Environmental hazards
OSHA defined hazards
Label elements
Hazard symbol
Signal word
Hazard statement
Precautionary statement
Prevention
Response
Storage
Disposal

Hazard(s) not otherwise

classified (HNOC)

Not

Not

Not

Drexel University

3141 Chestnut Street, Philadelphia, PA 19104 USA

A.J. Drexel Nanomaterials Institute

Yury Gogotsi

Tel +12158952000

gogotsi@drexel.edu

Same as above

Not classified.

None.

None.

The mixture does not meet the criteria for classification.

Observe good industrial hygiene practices.

Wash hands after handling.

Store away from incompatible materials.

None known.

None. classified.

classified.

classified.

Dispose of waste and residues in accordance with local authority requirements.

\section{Composition/information on ingredients}

Mixtures

\begin{tabular}{lcc} 
Chemical name & Common name and synonyms & CAS number \\
\hline WATER & $7732-18-5$ & $90-99$ \\
\hline Titanium carbide & $12363-89-2$ & $0.1-10$ \\
\hline Other components below reportable levels & $\leq .9$
\end{tabular}

\section{First-aid measures}

Inhalation

Skin contact

Eye contact

Ingestion

Most important symptoms/effects, acute and

delayed
Move to fresh air. Call a physician if symptoms develop or persist.

Wash off with soap and water. Get medical attention if irritation develops and persists.

Rinse with water. Get medical attention if irritation develops and persists.

Rinse mouth. Get medical attention if symptoms occur.

Direct contact with eyes may cause temporary irritation. 
Indication of immediate

medical attention and special

treatment needed

General information

\section{Fire-fighting measures}

Suitable extinguishing media

Unsuitable extinguishing

media

Specific hazards arising from the chemical

Special protective equipment and precautions for firefighters

Fire fighting equipment/instructions

Specific methods

General fire hazards

Treat symptomatically.

Ensure that medical personnel are aware of the material(s) involved, and take precautions to protect themselves.

Water fog. Foam. Dry chemical powder. Carbon dioxide (CO2).

Do not use water jet as an extinguisher, as this will spread the fire.

During fire, gases hazardous to health may be formed.

Self-contained breathing apparatus and full protective clothing must be worn in case of fire.

Move containers from fire area if you can do so without risk.

Use standard firefighting procedures and consider the hazards of other involved materials. No unusual fire or explosion hazards noted.

\section{Accidental release measures}

Personal precautions, protective equipment and emergency procedures

Methods and materials for containment and cleaning up

\section{Environmental precautions}

\section{Handling and storage}

Precautions for safe handling

Conditions for safe storage, including any incompatibilities
Keep unnecessary personnel away. For personal protection, see section 8 of the SDS.

The product is immiscible with water and will spread on the water surface.

Large Spills: Stop the flow of material, if this is without risk. Dike the spilled material, where this is possible. Absorb in vermiculite, dry sand or earth and place into containers. Following product recovery, flush area with water.

Small Spills: Wipe up with absorbent material (e.g. cloth, fleece). Clean surface thoroughly to remove residual contamination.

Never return spills to original containers for re-use. For waste disposal, see section 13 of the SDS. Avoid discharge into drains, water courses or onto the ground.

Avoid prolonged exposure. Observe good industrial hygiene practices.

Store in tightly closed container. Store away from incompatible materials (see Section 10 of the SDS).

\section{Exposure controls/personal protection}

\section{Occupational exposure limits}

The following constituents are the only constituents of the product which have a PEL, TLV or other recommended exposure limit. At this time, the other constituents have no known exposure limits.

Biological limit values

Appropriate engineering controls

Individual protection measures, such as personal protective equipment

Eye/face protection

Skin protection

Hand protection

Other

Respiratory protection

Thermal hazards

General hygiene

considerations

Wear suitable protective clothing.
No biological exposure limits noted for the ingredient(s).

Good general ventilation should be used. Ventilation rates should be matched to conditions. If applicable, use process enclosures, local exhaust ventilation, or other engineering controls to maintain airborne levels below recommended exposure limits. If exposure limits have not been established, maintain airborne levels to an acceptable level.

Wear safety glasses with side shields (or goggles).

Wear appropriate chemical resistant gloves.

In case of insufficient ventilation, wear suitable respiratory equipment.

Wear appropriate thermal protective clothing, when necessary.

Always observe good personal hygiene measures, such as washing after handling the material and before eating, drinking, and/or smoking. Routinely wash work clothing and protective equipment to remove contaminants. 


\section{Physical and chemical properties}

\section{Appearance}

$\begin{array}{ll}\text { Physical state } & \text { Liquid. } \\ \text { Form } & \text { Liquid } \\ \text { Color } & \text { Not aval }\end{array}$

Odor

Odor threshold

Not available.

pH

Not available.

$2.5-6$

Melting point/freezing point

Not available.

Initial boiling point and boiling Not available.

range

Flash point

Not available.

Evaporation rate

Not available.

Flammability (solid, gas) Not applicable.

Upper/lower flammability or explosive limits

Flammability limit - lower Not available.

(\%)

Flammability limit - upper Not available.

(\%)

Explosive limit - lower (\%) Not available.

Explosive limit - upper (\%) Not available.

Vapor pressure

Not available.

Vapor density

Not available.

Relative density

Not available.

Solubility(ies)

Solubility (water)

insoluble

Solubility (other)

insoluble

Partition coefficient

(n-octanol/water)

Auto-ignition temperature

Not available.

Decomposition temperature

Not available.

Not available.

Viscosity

$>0.5 \mathrm{mPa} \cdot \mathrm{s}$

Other information

Explosive properties

Not explosive.

Oxidizing properties Not oxidizing.

\section{Stability and reactivity}

\section{Reactivity}

Chemical stability

Possibility of hazardous

reactions

Conditions to avoid

Incompatible materials

Hazardous decomposition products
The product is stable and non-reactive under normal conditions of use, storage and transport. Material is stable under normal conditions.

No dangerous reaction known under conditions of normal use.

Contact with incompatible materials.

Strong oxidizing agents.

No hazardous decomposition products are known.

\section{Toxicological information}

Information on likely routes of exposure

Inhalation

Skin contact

Eye contact

Ingestion
Prolonged inhalation may be harmful.

Knowledge about health hazard is incomplete.

Knowledge about health hazard is incomplete.

Knowledge about health hazard is incomplete. 
Symptoms related to the

physical, chemical and

toxicological characteristics

Information on toxicological effects

\section{Acute toxicity}

Skin corrosion/irritation

Serious eye damage/eye

irritation

Respiratory or skin sensitization

Respiratory sensitization

Skin sensitization

Germ cell mutagenicity

Carcinogenicity

IARC Monographs. Overall Evaluation of Carcinogenicity

Not listed.

OSHA Specifically Regulated Substances (29 CFR 1910.1001-1053)

Not listed.

US. National Toxicology Program (NTP) Report on Carcinogens

Not listed.

Reproductive toxicity

Specific target organ toxicity single exposure

Specific target organ toxicity repeated exposure

Aspiration hazard

Chronic effects

\section{Ecological information}

Ecotoxicity

Persistence and degradability

Bioaccumulative potential

Mobility in soil

Other adverse effects
Due to partial or complete lack of data the classification is not possible.

Due to partial or complete lack of data the classification is not possible.

Due to partial or complete lack of data the classification is not possible.

Due to partial or complete lack of data the classification is not possible.

Prolonged inhalation may be harmful.

The product is not classified as environmentally hazardous. However, this does not exclude the possibility that large or frequent spills can have a harmful or damaging effect on the environment. No data is available on the degradability of any ingredients in the mixture.

No data available.

No data available.

No other adverse environmental effects (e.g. ozone depletion, photochemical ozone creation potential, endocrine disruption, global warming potential) are expected from this component.

\section{Disposal considerations}

Disposal instructions

Local disposal regulations

Hazardous waste code Waste from residues / unused
products

Contaminated packaging

\section{Transport information}

DOT

Not regulated as dangerous goods.

IATA

Not regulated as dangerous goods.

IMDG

Not regulated as dangerous goods.
Collect and reclaim or dispose in sealed containers at licensed waste disposal site.

Dispose in accordance with all applicable regulations.

The waste code should be assigned in discussion between the user, the producer and the waste disposal company.

Dispose of in accordance with local regulations. Empty containers or liners may retain some product residues. This material and its container must be disposed of in a safe manner (see: Disposal instructions).

Since emptied containers may retain product residue, follow label warnings even after container is emptied. Empty containers should be taken to an approved waste handling site for recycling or disposal. 
Annex II of MARPOL $73 / 78$ and

the IBC Code

\section{Regulatory information}

US federal regulations

This product is not known to be a "Hazardous Chemical" as defined by the OSHA Hazard Communication Standard, 29 CFR 1910.1200.

Toxic Substances Control Act (TSCA)

TSCA Section 12(b) Export Notification (40 CFR 707, Subpt. D)

Not regulated.

CERCLA Hazardous Substance List (40 CFR 302.4)

Not listed.

SARA 304 Emergency release notification

Not regulated.

OSHA Specifically Regulated Substances (29 CFR 1910.1001-1053)

Not listed.

Superfund Amendments and Reauthorization Act of 1986 (SARA)

SARA 302 Extremely hazardous substance

Not listed.

SARA 311/312 Hazardous No

chemical

SARA 313 (TRI reporting)

Not regulated.

Other federal regulations

Clean Air Act (CAA) Section 112 Hazardous Air Pollutants (HAPs) List Not regulated.

Clean Air Act (CAA) Section 112(r) Accidental Release Prevention (40 CFR 68.130) Not regulated.

Safe Drinking Water Act Contains component(s) regulated under the Safe Drinking Water Act. (SDWA)

\section{US state regulations}

\section{California Proposition 65}

California Safe Drinking Water and Toxic Enforcement Act of 1986 (Proposition 65): This material is not known to contain any chemicals currently listed as carcinogens or reproductive toxins. For more information go to www.P65Warnings.ca.gov.

International Inventories

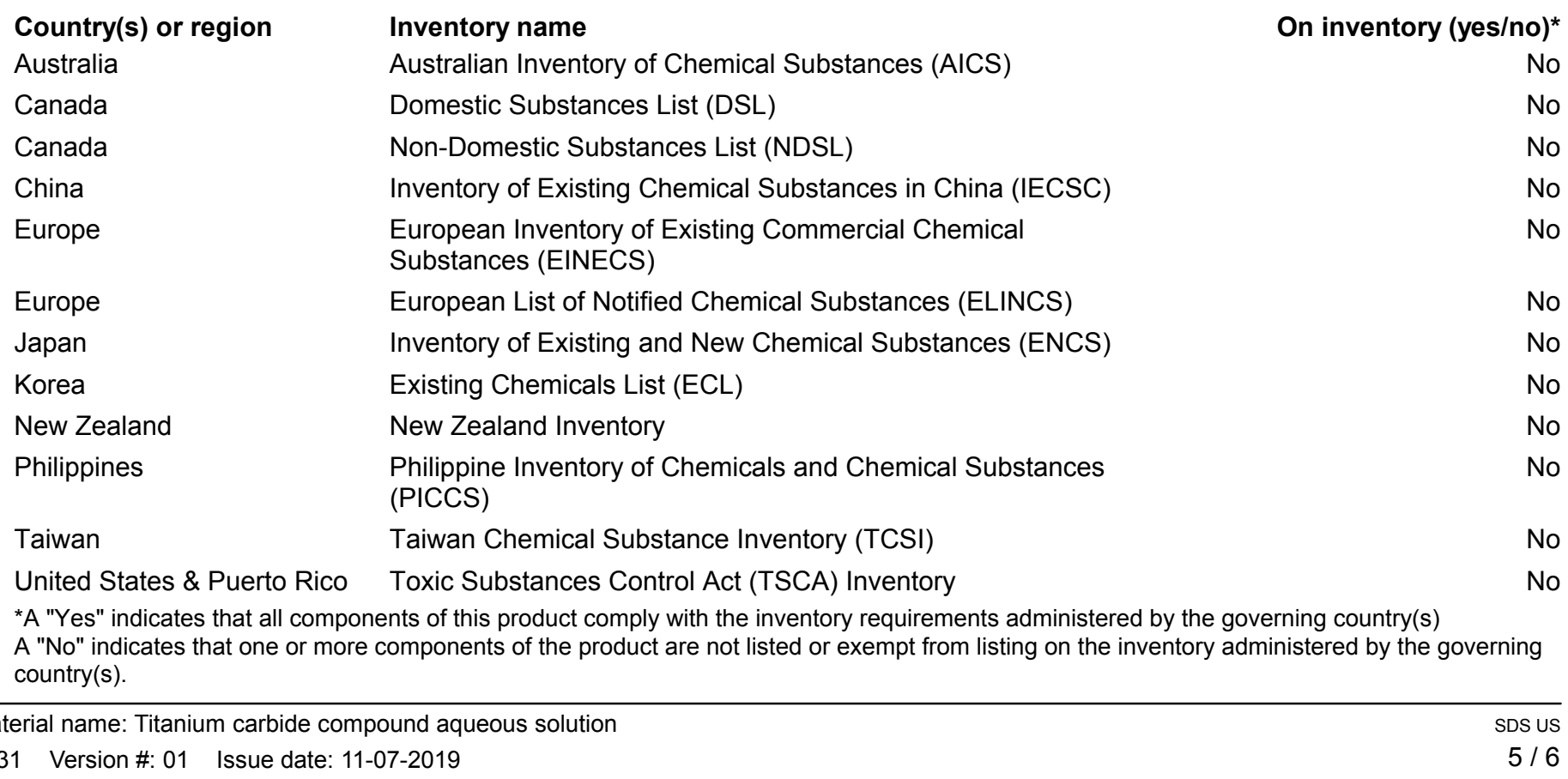




\section{Other information, including date of preparation or last revision}

Issue date

Version \#

Disclaimer

Revision information
11-07-2019

01

Murata Manufacturing Company, Ltd. Yasu Division cannot anticipate all conditions under which this information and its product, or the products of other manufacturers in combination with its product, may be used. It is the user's responsibility to ensure safe conditions for handling, storage and disposal of the product, and to assume liability for loss, injury, damage or expense due to improper use. The information in the sheet was written based on the best knowledge and experience currently available.

Product and Company Identification: Product and Company Identification Composition / Information on Ingredients: Ingredients

Physical \& Chemical Properties: Multiple Properties 